\title{
SPACE CHARGE LENS FOR FOCUSING NEGATIVE ION BEAMS
}

\author{
V.P.Goretsky, I.A.Soloshenko, A.I.Shchedrin \\ Institute of Physics of National Academy of Sciences of Ukraine, Kiev, Ukraine, \\ e-mail: solosh@marion.iop.kiev.ua, fax: +38(044)2651589, phone: +38(044)2650925
}

The idea of space charge lens for focusing negative ion beam is set forth. With the use of this lens focusing of $\mathrm{H}^{-}$ ion beam with $10 \mathrm{keV}$ energy and $30 \mathrm{~mA}$ current is realized. Focal length value of $\sim 12 \mathrm{~cm}$ is reached.

\section{Introduction}

The idea of the use of space charge fields for focusing positive ion beams for the first time was proposed by Gabor [1] and was further developed in proceedings of A.I.Morozov [2]. Efficiency of such lens has been confirmed in a number of experiments (e.g. in [3]). Negative space charge of the lens is formed by electrons, which are held by magnetic field. However, it should be noted that any design of space charge lens for positive ion beam cannot be used for negative ion beam because in the last case positive ions should be used for the focusing.

The idea of space charge lens for negative ion beam is based on the use of positive ions formed at gas ionization by the beam itself. That is, here the use of process, which is undesirable in case of focusing positive ion beams, is proposed. Since in ionization process the electrons are also formed, required positive space charge can be created only at condition of withdrawal these electrons by electric field. Large lifetime of positive ions in the system is ensured at that by their inertia. Particularly, the simplest focusing system may be represented by metal cylinder, which coaxially embraces the beam, and two electrodes at the ends of the cylinder. The last must be transparent for the beam, that is, should be grid or ring ones. In internal space of the cylinder required positive space charge will be created, if one will ensure withdrawal of the electrons by supplying positive potential to periphery electrodes with respect to central electrode. It should be noted that for efficient withdrawal of the electrons the potential should be applied at which extension of space charge layer is comparable with longitudinal dimension of the system. Such extended space charge layers in the system under study can be created already at potential difference between the lens electrodes of $100 \div 1000 \mathrm{~V}$ due to relatively low concentration of the beam plasma $\left(\sim 10^{8} \mathrm{~cm}^{-3}\right)$.

By supplying the gas immediately into the cylinder space required conditions for ionization may be created without essential increase of the pressure outside the lens.

\section{Estimation of focal power of space charge lens}

Obviously, proposed principle of the focusing can be realized only at condition when space charge of positive ions formed in result of gas ionization exceeds space charge of the beam ions. It applies limitation on low bound for the pressure of associated gas. The value of required gas concentration can be determined from the balance equation for positive ions formation and leaving, and is given by formula:

$$
\mathrm{n}_{\mathrm{a}} \geq \frac{2 \mathrm{v}_{+}}{\mathrm{v}_{-} \sigma_{\mathrm{i}} \mathrm{r}_{0}}
$$

where $n_{a}$ is gas concentration; $\sigma_{i}$ is cross section of ionization of the gas particles by the beam ions; $\mathrm{v}_{+}, \mathrm{v}_{-}$are velocities of leaving ions and the beam particles, respectively; $r_{0}$ is the beam radius [4]. In regime of free propagation of the beam, at fulfilling the condition (1), the beam potential with respect to periphery is positive, and its value is determined by average thermal energy of the plasma electrons which, in turn, is defined by Coulomb collisions with the beam ions. Focusing fields, which arise at that in the beam with concentration of $\sim 10^{8} \mathrm{~cm}^{-3}$, possess strength just several $\mathrm{V} / \mathrm{cm}$ and thus can be used just for improvement of transportation of weakly divergent beams.

Usually for focusing the beams extracted from the single aperture the lenses with focal length $10-20 \mathrm{~cm}$ are necessary which requires radial fields $\sim 100 \mathrm{~V} / \mathrm{cm}$. Such fields in the system under study may be achieved only at condition of practically total withdrawal of the electrons from the focusing region. At fulfilling this condition the electrons space charge may be neglected, and focusing features of the lens can be estimated comparatively easily.

As initial equations for calculation of focusing field we use the continuity equation for positive ions, Poisson equation for the potential and the equation for positive particle motion in radial electric field:

$$
\begin{aligned}
& \frac{1}{\mathrm{r}} \frac{\partial}{\partial \mathrm{r}} \mathrm{rj}_{+}=\mathrm{en}_{-} \mathrm{n}_{\mathrm{a}} \sigma_{\mathrm{i}} \mathrm{V}_{-}=\frac{\mathrm{j}_{-}}{\lambda_{\mathrm{i}}} \\
& \frac{1}{\mathrm{r}} \frac{\partial}{\partial \mathrm{r}} \mathrm{r} \frac{\partial}{\partial \mathrm{r}} \varphi=-4 \pi \mathrm{e}\left(\mathrm{n}_{+}-\mathrm{n}_{-}\right) \\
& \mathrm{V}_{+}\left(\mathrm{r}_{\mathrm{i}}, \mathrm{r}\right)=\sqrt{\frac{2 \mathrm{e}\left(\varphi\left(\mathrm{r}_{\mathrm{i}}\right)-\varphi(\mathrm{r})\right)}{\mathrm{m}_{+}}}
\end{aligned}
$$

Here $\lambda_{i}$ is path length of the beam particle till gas ionization; $\mathrm{V}_{+}\left(\mathrm{r}_{\mathrm{i}}, \mathrm{r}\right)$ is velocity of positive particle formed at $r_{i}$ point and reached $r$ point.

From (2) and (4) in assumption of uniform distribution of positive ions concentration along the radius one can determine (in regime of strong overcompensation of the beam space charge) that radial fall of the potential is the following:

$$
\varphi_{\mathrm{a}} \approx\left(\frac{\mathrm{I}_{-} \mathrm{r}_{0}}{\lambda_{\mathrm{i}} \sqrt{2 \mathrm{e} / \mathrm{m}_{+}}}\right)^{\frac{2}{3}} \text {. }
$$


In opposite case, which is achieved by the beam current increase and corresponds to quasineutral regime, the potential fall is given by:

$$
\varphi_{\mathrm{a}} \approx \frac{\mathrm{r}_{0}^{2} \mathrm{~m}_{+}}{\lambda_{\mathrm{i}}^{2} \mathrm{~m}_{-}} \mathrm{U}_{-} .
$$

Here $I_{-}$and $U_{-}$are current and energy of negative ion beam. It follows from equations (5) and (6) that at the increase of beam current radial fall of the potential $\varphi_{\mathrm{a}}$ grows up at first, and after that reaches its maximum value which is independent on the beam current value.

It follows from the expressions given above that for the beam of hydrogen negative ions with energy $\sim 10 \mathrm{kEv}$ in the lens with dimensions $\sim 10 \mathrm{~cm}$ at gas (argon, krypton, xenon) pressure $\sim 10^{-3}$ Torr actually achievable radial fields exceed $100 \mathrm{~V} / \mathrm{cm}$.

Using methods described in [5] it is easy to obtain expression for focal power of the lens:

$$
\frac{1}{\mathrm{f}}=\frac{1}{\sqrt{\mathrm{U}_{-}(0)}} \int_{0}^{\mathrm{L}} \frac{\varphi_{\mathrm{a}} \mathrm{dz}}{\mathrm{r}_{0}^{2} \sqrt{\mathrm{U}_{-}(\mathrm{z})}} \approx \frac{\varphi_{\mathrm{a}} \mathrm{L}}{\mathrm{U}_{-} \mathrm{r}_{0}^{2}} .
$$

Substituting $\varphi_{\mathrm{a}}$ in (7) by expressions from (5) and (6) we obtain for large current regime (quasineutral regime):

$$
\frac{1}{\mathrm{f}}=\frac{\mathrm{Lm}_{+}}{\lambda_{\mathrm{i}}^{2} \mathrm{~m}_{-}},
$$

and for small current regime:

$$
\frac{1}{\mathrm{f}}=\left(\frac{\Pi}{9 \pi}\right)^{\frac{2}{3}}\left(\frac{\mathrm{m}_{+}}{\mathrm{m}_{-}}\right)^{\frac{1}{3}} \frac{\mathrm{L}}{\left(\lambda_{\mathrm{i}} \mathrm{r}_{0}^{2}\right)^{\frac{2}{3}}}=\left(\frac{\mathrm{j}_{-} \pi}{\lambda_{\mathrm{i}} \sqrt{2 \mathrm{e} / \mathrm{m}_{+}}}\right)^{\frac{2}{3}} \frac{\mathrm{L}}{\mathrm{U}_{-}} .
$$

It follows from (8) that at lens parameters given above required focal length value $\sim 10 \mathrm{~cm}$ is achieved, and the losses of negative ions at the beam current density $>45 \mathrm{~mA} / \mathrm{cm}^{2}$ are less than $10 \%$.

\section{EXPERIMENTAL STUDY OF $\mathrm{H}^{-}$ION BEAM FOCUSING BY SPACE CHARGE LENS}

The researches have been performed at the setup shown schematically on Fig.1. The beam of $\mathrm{H}^{-}$negative ions with current $\sim 10 \div 30 \mathrm{~mA}$ and energy $\sim 10 \mathrm{kEv}$ was extracted from the source of surface-plasma type 1 . Beam formation and turn were accomplished by magnetic field $\sim 2 \mathrm{kGs}$ by means of magnets 2 . The beam current was measured by collector 7 with diameter $\sim 10 \mathrm{~cm}$, current density was measured by collector 6 with $2 \mathrm{~cm}$ diameter. Space charge lens was placed at distance $\sim 20 \mathrm{~cm}$ from emission slit of the source. Distance from output plane of the lens till the collector comprised $\sim 30 \mathrm{~cm}$. With such geometry of the system minimum beam radius should be observed at distance $12 \mathrm{~cm}$. Lens design was the following: inside grounded cylindrical cabinet 3 made from stainless steel with $10 \mathrm{~cm}$ external diameter, $13 \mathrm{~cm}$ length, input and output apertures with $5 \mathrm{~cm}$ diameter, metal cylinder 5 with $7 \mathrm{~cm}$ diameter and $10 \mathrm{~cm}$ length was placed. Presence of the last unit allowed maintenance of uniform distribution of the gas pressure inside the lens. Its potential was hold either at ground level, or at level of electrode 4 potential (it had no essential influence on focusing features of the lens). Cylindrical electrode 4 made from stainless steel grid with $5 \mathrm{~cm}$ diameter and $10 \mathrm{~cm}$ length was mounted inside electrode 5 by means of dielectric rings at the ends. During the experiment the potential of electrode 4 could be varied in range from 0 down to $-1500 \mathrm{~V}$. At outer side of the cabinet two sockets were placed; one of those served for supply of working gas, and another was used for measurement of the pressure in lens region. Pressure in the lens section and in the beam drift chamber differed at that for more than one order of magnitude. Argon, krypton and xenon were used as working gases. Choice of those gases was due to, at first, comparatively high values of ionization cross sections and, at second, comparatively large inertia of positive ions formed in result of ionization. Both factors facilitated creation of the conditions required for efficient focusing of negative ion beam.

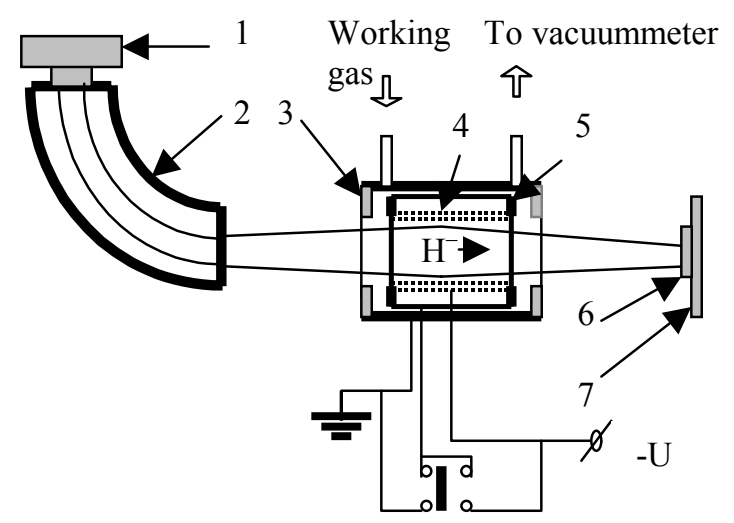

\section{Fig.1. Schematic drawing of the experiment}

Before proceeding to description of the experimental results, let us estimate critical pressure, in excess of which the charge formed due to positive ions created during the gas ionization starts to overcome essentially negative space charge of negative ion beam. For the estimation let us suggest that average energy of ions created in result of the gas ionization by the beam comprises $\sim 1 \mathrm{eV}$. Then from expression (1) and known from proceeding [6] values of ionization cross section for the beam with parameters given above we obtain: $\mathrm{P}_{\mathrm{o}} \sim 1.5 \cdot 10^{-4}$ Torr for argon, $\mathrm{P}_{\mathrm{o}} \sim 4 \cdot 10^{-5}$ Torr for krypton, $\mathrm{P}_{\mathrm{o}} \sim 6 \cdot 10^{-5}$ Torr for xenon. At pressure above $\mathrm{P}_{\mathrm{o}}$ the rate of positive ions creation is proportional to the pressure, however, density of positive ions charge grows up at that slightly slower due to the increase of the rate of their leaving along the radius at the expense of radial potential fall growth.

As it was shown by the experiments, in agreement with the estimation for the pressures higher than critical one the effect of negative ion beam focusing is observed. The most clear demonstration of focusing features of the lens is exhibited in Fig. 2 which presents the dependencies of the beam compression degree on potential difference between the lens electrodes (grounded cabinet 3 and grid electrode 4) obtained at various pressures of argon. The beam compression 
degree means here the ratio of maximum current density with optimal potentials applied to the electrodes to that without applying the potentials. Behavior of the curves is in agreement with qualitative considerations given above. At first the beam is compressed with the growth of potential difference, but starting from $\mathrm{V} \sim 200 \mathrm{~V}$ all curves reach the saturation. It is explained by the fact that at $\mathrm{V}>200 \mathrm{~V}$ electrons density in the beam becomes inessential and radial fall of the potential is mainly due to positive ions and beam ions. At the pressure increase the rate of compression degree growth increases which is due to the growth of positive space charge. At that maximum compression degree increases at first (curves
1-3), and decreases in subsequent (curves 4-6) which can be explained by the beam overfocusing. The last circumstance was confirmed by special experiments in which metal plate with two holes was placed at $4 \mathrm{~cm}$ distance from the lens output aperture, and at distance of $22 \mathrm{~cm}$ luminescent screen was situated which served for observation of images of the holes. At optimal potential difference at the lens electrodes xenon pressure increase results at first to converging of the holes images, and at high enough pressure these images join together, and after that diverge in direction opposite to initial one with respect to the center thus giving unambiguous evidence of the beam overfocusing.

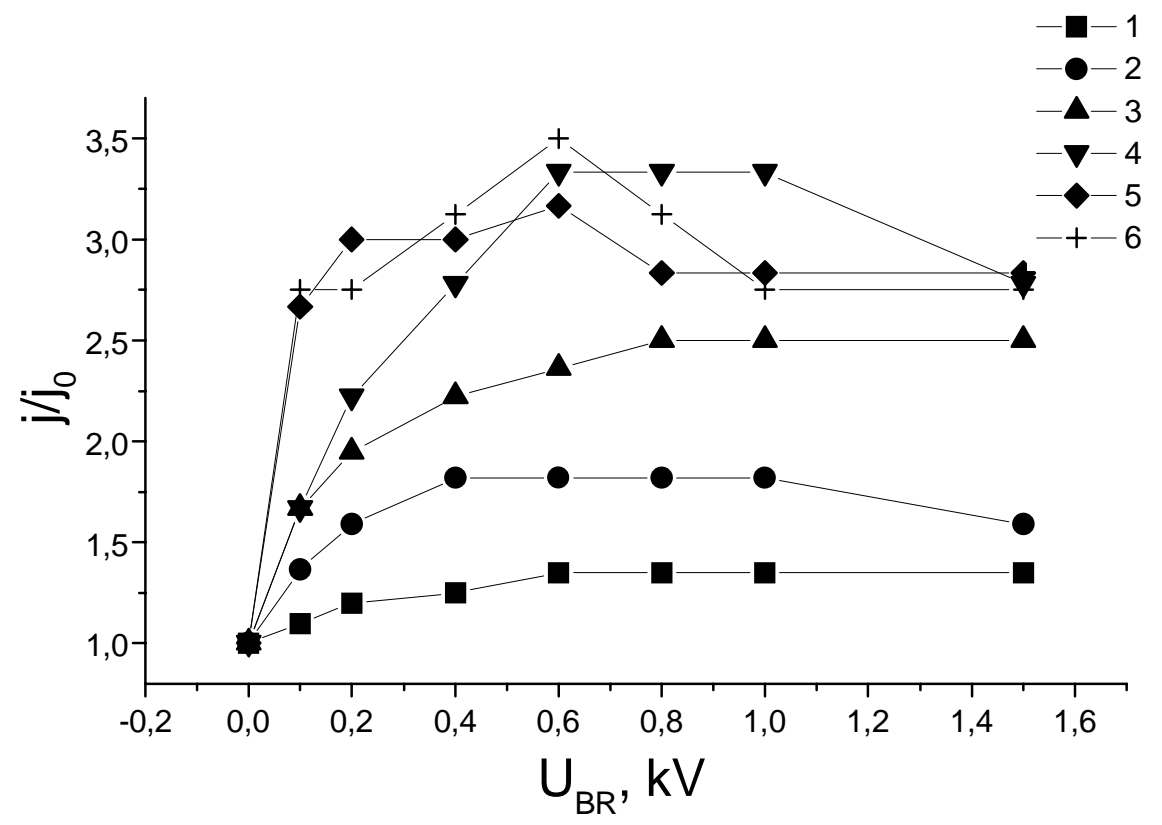

Fig.2. Dependence of hydrogen negative ion beam compression $j / j_{0}$ on negative potential of braking cylinder $U_{B R}$. Argon pressure (Torr) in the lens is: $3 \cdot 10^{-4}(1)$, $7.6 \cdot 10^{-4}(2), 1.5 \cdot 10^{-3}(3), 2.2 \cdot 10^{-3}(4), 3.6 \cdot 10^{-3}(5), 6.4 \cdot 10^{-3}(6)$

And, finally, the fact that maximum compression degree at given system geometry corresponds to focal length $\sim 12 \mathrm{~cm}$ can be used for comparison of the experimental results with the calculations. Let us choose for the comparison the case of argon use in which optimal pressure comprises $\sim 3 \cdot 10^{-3}$ Torr. Accordingly to (9), at beam current of $15 \mathrm{~mA}$ calculated value of focal length is $\sim 20 \mathrm{~cm}$. This value is close enough to actually measured one ( $\mathrm{f} \sim 12 \mathrm{~cm}$ ).

Thus, in current proceeding the method of negative ion beam focusing by means of space charge lens is proposed and realized. This method possesses practical interest for the beams with comparatively large current density in which acquirement of big enough positive space charge can be reached without significant losses of the beam ions due to overcharging.

\section{References}

1. D.Gabor. Space Charge Lense for Focusing Ions Beams. // Nature. 1947, 160, p.89-90.

2. A.I.Morozov. Focusing of cold quasineutral beams in electromagnetic fields. // Reports of Acad. of Sciences of the USSR. 1965, 163, p.1363-1368.

3. A.A.Goncharov, A.N.Dobrovolsky, A.N.Kotsarenko, A.I.Morozov, I.M.Protsenko. Static and dynamic properties of high-current plasma lens. // Fiz. Plasmy. 1994, 20, N.5, p.499-505.

4. M.D.Gabovich, L.S.Simonenko, I.A.Soloshenko. Compensation of the space charge of intensive negative ion beam. // Zh.Tekh.Fiz. 1978, 48, N.7, p.1389-1393.

5. V.M.Kelman, S.Ya.Yavor. Electron Optics. Moscow: USSR Academy of Science Publishing, 1959.

6. Ya.M.Fogel, A.G.Koval, Yu.Z.Levchenko. Gas ionization by negative ions. // Zh.Eksp.Teor.Fiz. 1960, 38, N.4, p.1053-1060. 